\title{
TRSTMH
}

\section{A pilot study using wearable global position system (GPS) data loggers to compare water contact levels: Schistosoma haematobium infection in pre-school-aged children (PSAC) and their mothers at Barombi Kotto, Cameroon \\ --Manuscript Draft--}

\begin{tabular}{|l|l|}
\hline Article Type: & Short Communication \\
\hline Full Title: & $\begin{array}{l}\text { A pilot study using wearable global position system (GPS) data loggers to compare } \\
\text { water contact levels: Schistosoma haematobium infection in pre-school-aged children } \\
\text { (PSAC) and their mothers at Barombi Kotto, Cameroon }\end{array}$ \\
\hline Abstract: & $\begin{array}{l}\text { Barombi Kotto, Cameroon serves as a reference location for assessing intervention } \\
\text { strategies against Schistosoma haematobium. As part of a pilot study, the whole } \\
\text { community was treated with praziquantel, inclusive of pre-school-aged children (PSAC) } \\
\text { and their mothers. One year later egg-patent infections were reassessed and water } \\
\text { contact patterns of } 12 \text { pairs of PSAC and their mother were measured with global } \\
\text { position system (GPS) dataloggers. A substantial reduction in general infection } \\
\text { prevalence, from 44.8\% to 11.7 \%, was observed but certain PSAC and mothers } \\
\text { continued to have egg-patent infections. Analysis of GPS data demonstrated similar } \\
\text { water contact levels between child and mother groups, although certain individuals } \\
\text { were numerical outliers. This study shows the potential of GPS dataloggers to clarify } \\
\text { the at-risk status of PSAC and mothers. }\end{array}$ \\
\hline TRSTMH-D-18-00041R1 \\
\hline Manuscript Number: & Grace MacKlin, MSc \\
\hline Order of Authors: & Michelle Stanton, PhD \\
\hline Louis Albert Tchuem-Tchuente \\
\hline John Russell Stothard \\
\hline
\end{tabular}




\section{A pilot study using wearable global position system (GPS) dataloggers to compare water} contact levels: Schistosoma haematobium infection in pre-school-aged children (PSAC) and their mothers at Barombi Kotto, Cameroon

Grace Macklin $^{\mathrm{a},+}$, Michelle C. Stanton ${ }^{\mathrm{b}}$, Louis Albert Tchuem-Tchuentéc, J. Russell Stotharda, ${ }^{\mathrm{a}}{ }^{*}$ ${ }^{a}$ Department of Parasitology, Liverpool School of Tropical Medicine, Liverpool, UK; ${ }^{b}$ Faculty of Health and Medicine, Lancaster University, Lancaster, UK; ${ }^{\mathrm{C}}$ Centre for Schistosomiasis \& Parasitology, Yaoundé, Cameroon

+ present address: London School of Hygiene and Tropical Medicine, Keppel Street, London WC1E 7HT, UK;

* Corresponding author: Tel: +44 151 7053724; E-mail: russell.stothard@lstmed.ac.uk

\section{Abstract}

Barombi Kotto, Cameroon serves as a reference location for assessing intervention strategies against Schistosoma haematobium. As part of a pilot study, the whole community was treated with praziquantel, inclusive of pre-school-aged children (PSAC) and their mothers. One year later egg-patent infections were reassessed and water contact patterns of 12 pairs of PSAC and their mother were measured with global position system (GPS) dataloggers. A substantial reduction in general infection prevalence, from $44.8 \%$ to $11.7 \%$, was observed but certain PSAC and mothers continued to have egg-patent infections. Analysis of GPS data demonstrated similar water contact levels between child and mother groups, although certain individuals were numerical outliers. This study shows the potential of GPS dataloggers to clarify the at-risk status of PSAC and mothers.

\section{Keywords}

Urogenital schistosomiasis, i-gotU, paediatric schistosomiasis, female genital schistosomiasis, praziquantel 


\section{Introduction}

Urogenital schistosomiasis is an important waterborne disease, caused by infection with the blood fluke Schistosoma haematobium, and common in many parts of sub-Saharan Africa (1). In Cameroon, for example, there is a national control programme active in the distribution of praziquantel (PZQ) to school-aged children (SAC) $(2,3)$. However, in the move towards local interruption of schistosome transmission, the programme is developing new tactics of control (4) and has benefited from recent bilateral support from China in snail control and environmental surveillance (5), as well as from UK to expand access of interventions (6).

Overlooked for too long, expanding access of PZQ to pre-school-aged children (PSAC) and their mothers is attracting increasing attention $(7,8)$. It has been shown elsewhere that these groups can be patently infected (9-12) and alongside SAC, may contribute towards schistosome transmission but their water contact(s) is rarely measured and hence the role of PSAC in sustaining local transmission remains speculative $(13,14)$. As a pilot investigation of expanded access to praziquantel treatment, in June 2016 Campbell et al. undertook a detailed cross-sectional epidemiological and malacological survey at Barombi Kotto, Cameroon (15). Barombi Kotto is well-known crater lake and is of significant international interest as a longstanding focus of urogenital schistosomiasis (16-18). Before treating all community members with PZQ, Campbell et al. noted that a quarter of PSAC had egg-patent infections. Furthermore, adult women had raised signs and symptoms of female genital schistosomiasis (FGS), the latter is of growing international concern $(13,19)$. Environmental water contact is very common across the community, for example, bathing, washing and other domestic chores are typically performed on the immediate shoreline of the island while potable water is collected in plastic containers from a local stream which is only accessible by canoe (15). The level of environmental water contact, however, on the immediate lake shoreline of both PSAC and their mothers remained to be determined and compared.

To shed fresh light on the at-risk status of PSAC and their mothers, using wearable global position system (GPS) dataloggers, we attempted to measure and compare the water contact patterns of PSAC and their mothers $(20,21)$. Furthermore, we hoped to pinpoint water contact sites, measuring putative immersion times, on the Barombi Kotto crater lake shoreline as baseline information for future interventions. 
Methods \& Materials

64

65

66

67

68

69

70

71

72

\section{Study location and parasitological examination}

This parasitological resurvey and GPS study was conducted in June 2017 in the community on the central island of Barombi Kotto crater lake, where some 375 people are permanent residents. Study protocols were approved by the Liverpool School of Tropical Medicine Research Ethics Committee and the Cameroon National Ethical Committee of Research for Human Health. Participation involved obtaining written informed consent from mothers and their PSAC before deployment of the GPS datalogger. A total of 179 individuals (20 PSAC, 55 SAC and 104 adults) underwent a parasitological reinspection where each provided a $10 \mathrm{ml}$ urine sample which was filtered and stained with Lugol's iodine to visualise S. haematobium eggs by microscopy as described previously (15). All participants found infected with schistosomiasis were offered and observed to take praziquantel treatment $(40 \mathrm{mg} / \mathrm{kg}$ )

\section{Water exposure assessment}

A subset of 12 mothers and PSAC pairs were randomly selected, then 6 pairs were assigned into two groups to wear the GPS dataloggers (i-gotU-120, Mobile Action, UK; dimension 44.5 x $28.5 \times 13 \mathrm{~mm}$, weight $20 \mathrm{~g}$ ) over a 48-hour period on two occasions. The dataloggers were worn on the arm or wrist using a custom made elastic strap (20). The dataloggers were configured to record GPS location and velocity at 1 minute intervals during waking hours (05:00-21:00). Analysis of GPS data was conducted in QGIS (22) and filtered per the velocity filtering method (23). A zone was developed around the lakeshore of the island, 10m into the lake and $5 \mathrm{~m}$ into the shore, a conservative assessment of the positional accuracy of the igotU-120 dataloggers based on previous observations (24). A water contact event was defined as a GPS location recording within a defined lakeshore geospatial buffer zone around the island circumference: a conservative assessment of the positional accuracy of the i-gotU dataloggers (24). As the GPS loggers recorded location at 1 minute intervals, each water contact event is analogous to 1 minute spent in the geospatial buffer zone and could be tallied and compared between individuals and groups. 


\section{Statistical analysis}

Statistical analysis was performed using the $\mathrm{R}$ statistical software (25). Prevalence of schistosomiasis was calculated with $95 \%$ binomial confidence intervals (95\% Cls) with correction for samples of $n<30$. The track logs of each GPS unit were plotted and overlaid against a base map of Barombi Kotto shoreline to identify travel patterns on and off the island.

\section{Results and Discussion}

\section{$<$ please insert table 1 here $>$}

The characteristics of the study population and infection status is shown in Table 1. In June 2017, the overall prevalence of egg-patent infection was $11.7 \%$ (95\% Cl 7.0 - 17.0) with only 1 infection of heavy intensity encountered albeit in a PSAC. The epidemiological survey undertaken by Campbell et al. one year previously observed a much higher egg-patent prevalence of $40.1 \%$ (24.6\% in PSAC, $51.3 \%$ in SAC and $44.9 \%$ in adults). The overall reduction across all demographic groups, see Table 1 , is most likely due to the community-wide PZQ treatment.

\section{<please insert Figure 1 here $>$}

Twelve PSAC and mother pairs were randomly selected and assigned into two groups of 6 pairs, to wear the GPS dataloggers on two locations. In total, there were 3 individuals with egg-patent infections ( 2 mothers and 1 PSAC) and their intensities of egg-patent infections against the sampled population is shown in Figure 1A. The water contact levels of the 12 mother and child pairs for the 48 hour period is shown in Figure 1B. This documents a high level of water contact events (reaching up to 166 water contact events in 24 hours) in both mothers and PSAC on the Barombi Kotto shoreline. As data points were recorded at 1 minute intervals, the number of water contact events can be interpreted as the time spent in water contact which would likely positively correlate with actual bodily immersion or skin contact with lake shore water.

The average number of water contact events in 24 hours were 27.4 [95\% Cls: -1.3 , 56.1] for mothers and 14.1 [95\% Cls: 8.5 ,19.7] for PSAC, with no significant difference 
between the two groups, Figure 1B. These findings have important implications in that water contact levels of PSAC should not to be overlooked and follow similar levels to that observed in Uganda (20). In Uganda PSAC were observed to spend on average half an hour on the shoreline of Lake Albert and were clearly shown to be an at-risk vulnerable group not only to first infection but also re-infection (10), as evidenced here in Barombi Kotto. Our study shows the potential of GPS data logging technology to clarify their at-risk status which should assist in better infection surveillance and control of urogenital schistosomiasis in general as well as for regular access to treatment with the soon to be deployed paediatric PZQ formulation (12). Upon more detailed inspection of individual water contact patterns, whilst our GPS sample of two infected mothers ("M1", "M2") and one infected PSAC ("X4") was too small to determine a precise relationship between water exposure and infection status, two of these individuals were clear numerical outliers in terms of their water contact(s) $(4.6,166.1$, and 31.8 for "M1", "M2" and "X4", respectively). The latter two lying far outside the confidence intervals for PSAC and mother averages. This demonstrates the importance of individual variation in exposure and likely environmental contamination, Figure 1B. Indeed, it is very plausible that the water contact behaviours of " $\mathrm{M} 2$ " and " $\mathrm{X} 4$ " could classify them as 'raisedspreaders' who should be specifically targeted for increased frequency of treatment(s) alongside behavioural change interventions. It remains to be seen if these individuals play more pivotal roles than others in facilitating and sustaining local transmission of schistosomes.

Another interesting facet revealed by the GPS dataloggers is the similar geospatial pattern of water contact between the two groups which illustrate that PSAC frequently accompany their mothers to the same locations, Figure $C$. This is also consistent with other studies using questionnaires $(13,21)$. Furthermore, observed water contacts were largely coclustered on the South-West lakeshore of the island, notably an area where activities of the 3 infected cases were concentrated. Micro-spatial heterogeneity of schistosome transmission has been described elsewhere $(26,27)$ and is further evidenced here, Figure 1D. In the future context of interruption of schistosome transmission $(14,27)$, as a cost-effective measure, it would be sensible to apply focal molluscicides at this location rather than elsewhere, to have highest impact upon removal of infected snail hosts. water exposure in PSAC and their mothers and directly compare environmental risk of 
schistosomiasis exposure. We suggest that in future the water contact levels of these two demographic groups should further investigated. The wearable GPS technology is also of value to identify putative transmission foci for spatial targeting of interventions.

161

162

163

\section{Authors Contributions}

JRS and MS conceived the study; GM, MS, LATT and JRS designed the study protocol; GM carried out the field work; GM and MS carried out the analysis and interpretation of these data. GM and JRS drafted the manuscript with LATT and MS critically revising. All authors read and approved the final manuscript.

\section{Funding}

GM is in receipt of a Scholarship from the Medical Research Council, United Kingdom. This study was additionally funded as part of the COUNTDOWN research programme consortium, by the Research and Evidence Division of the Department for International Development, UK. The funders had no role in study design, decision to publish, or preparation of the manuscript.

\section{Acknowledgments}

We are grateful to Drs James LaCourse and Martyn Stewart, LSTM who helped with local fieldwork in Cameroon, as well as, the local community and field team volunteers at Barombi Kotto.

\section{Competing interests}

None declared

\section{Ethical approval}

The study was approved by the Liverpool School of Tropical Medicine and the Cameroon National Ethical Committee of Research for Human Health.

\section{References}

1. Colley DG, Bustinduy AL, Secor WE, et al.; Human schistosomiasis. Lancet 2014;383(9936):2253-64. doi: 10.1016/S0140-6736(13)61949-2.

2. Tchuente LAT, N'Goran EK; Schistosomiasis and soil-transmitted helminthiasis control in Cameroon and Cote d'Ivoire: implementing control on a limited budget. Parasitology 2009;136(13):1739-1745. doi: 10.1017/s0031182009005988.

3. Tchuente LAT, Noumedem CD, Ngassam P, et al.; Mapping of schistosomiasis and soil-transmitted helminthiasis in the regions of Littoral, North-West, South and South-West 
Cameroon and recommendations for treatment. Bmc Infectious Diseases 2013;13. doi: 10.1186/1471-2334-13-602.

194 4. Tchuente LAT, Rollinson D, Stothard JR, et al.; Moving from control to elimination of schistosomiasis in sub-Saharan Africa: time to change and adapt strategies. Infectious Diseases of Poverty 2017;6. doi: 10.1186/s40249-017-0256-8. 5. $\mathrm{Xu} \mathrm{J,} \mathrm{Yu} \mathrm{Q,} \mathrm{Tchuente} \mathrm{LAT,} \mathrm{et} \mathrm{al.;} \mathrm{Enhancing} \mathrm{collaboration} \mathrm{between} \mathrm{China} \mathrm{and} \mathrm{African}$ countries for schistosomiasis control. Lancet Infectious Diseases 2016;16(3):376-383. doi: 10.1016/s1473-3099(15)00360-6.

200

201

6. Stothard JR, Kabatereine NB, Archer J, et al.; A centenary of Robert T. Leiper's lasting legacy on schistosomiasis and a COUNTDOWN on control of neglected tropical diseases. Parasitology 2017;144(12):1602-1612. doi: 10.1017/s0031182016000998.

7. Bustinduy AL, Friedman JF, Kjetland EF, et al.; Expanding Praziquantel (PZQ) Access beyond Mass Drug Administration Programs: Paving a Way Forward for a Pediatric PZQ Formulation for Schistosomiasis. Plos Neglected Tropical Diseases 2016;10(9). doi: 10.1371/journal.pntd.0004946.

8. Lo NC, Addiss DG, Hotez PJ, et al.; A call to strengthen the global strategy against schistosomiasis and soil-transmitted helminthiasis: the time is now. Lancet Infectious Diseases 2017;17(2):E64-E69. doi: 10.1016/s1473-3099(16)30535-7.

210 9. Bustinduy AL, Stothard JR, Friedman JF; Paediatric and maternal schistosomiasis: shifting the paradigms. British Medical Bulletin 2017;123(1):115-125. doi: $10.1093 / \mathrm{bmb} / \mathrm{ldx} 028$.

10. Mduluza T, Mutapi F; Putting the treatment of paediatric schistosomiasis into context. Infectious Diseases of Poverty 2017;6. doi: 10.1186/s40249-017-0300-8.

11. Stothard JR, Sousa-Figueiredo JC, Betson M, et al.; Schistosomiasis in African infants and preschool children: let them now be treated! Trends in Parasitology 2013;29(4):197205. doi: 10.1016/j.pt.2013.02.001.

218 12. Reinhard-Rupp J, Klohe K; Developing a comprehensive response for treatment of children under 6 years of age with schistosomiasis: research and development of a pediatric formulation of praziquantel. Infectious Diseases of Poverty 2017;6. doi: 10.1186/s40249017-0336-9.

222 13. Poole H, Terlouw DJ, Naunje A, et al.; Schistosomiasis in pre-school-age children and 223 their mothers in Chikhwawa district, Malawi with notes on characterization of schistosomes and snails. Parasites \& Vectors 2014;7. doi: 10.1186/1756-3305-7-153.

14. Stothard JR, Campbell SJ, Osei-Atweneboana MY, et al.; Towards interruption of schistosomiasis transmission in sub-Saharan Africa: developing an appropriate environmental surveillance framework to guide and to support 'end game' interventions. Infectious Diseases of Poverty 2017;6. doi: 10.1186/s40249-016-0215-9.

15. Campbell SJ, Stothard JR, O'Halloran F, et al.; Urogenital schistosomiasis and soiltransmitted helminthiasis (STH) in Cameroon: An epidemiological update at Barombi Mbo and Barombi Kotto crater lakes assessing prospects for intensified control interventions. Infectious Diseases of Poverty 2017;6. doi: 10.1186/s40249-017-0264-8. 16. Duke BOL, Moore PJ; Use of a molluscicide in conjunction with chemotherapy to control Schistosoma haematobium at Barombi Lake Foci in Cameroon. 3. Conclusions and costs. Tropenmedizin Und Parasitologie 1976;27(4):505-508.

17. Gonsufotsin J, Tagnizukam D, Moyousomo R, et al.; Ultrasonographic study of urological lesions of urinary bilhariasis in children in Barombi Kotto (Cameroon). Semaine Des Hopitaux 1990;66(13):680-684. 
18. Somo RM, Zukam DT, Kouamouo J, et al.; An epidemiological and radiological study of vesical schistosomiasis foci of the Barombi Lakes, Meme Division, Cameroon. Bulletin De La Societe De Pathologie Exotique 1987;80(5):813-822.

19. Christinet V, Lazdins-Helds JK, Stothard JR, et al.; Female genital schistosomiasis (FGS): from case reports to a call for concerted action against this neglected gynaecological disease. International Journal for Parasitology 2016;46(7):395-404. doi: 10.1016/j.ijpara.2016.02.006.

20. Seto EYW, Sousa-Figueiredo JC, Betson M, et al.; Patterns of intestinal schistosomiasis among mothers and young children from Lake Albert, Uganda: water contact and social networks inferred from wearable global positioning system dataloggers. Geospatial Health 2012;7(1):1-13.

21. Stothard JR, Sousa-Figueiredo JC, Betson M, et al.; Investigating the spatial microepidemiology of diseases within a point-prevalence sample: a field applicable method for rapid mapping of households using low-cost GPS-dataloggers. Transactions of the Royal Society of Tropical Medicine and Hygiene 2011;105(9):500-506. doi: 10.1016/j.trstmh.2011.05.007.

22. QGIS Development Team; QGIS Geographic Information System. Open Source Geospatial Foundation, 2009.

257

258

259 23. Seto EYW, Knapp F, Zhong B, et al.; The use of a vest equipped with a global positioning system to assess water-contact patterns associated with schistosomiasis. Geospatial Health 2007;1(2):233-241. doi: 10.4081/gh.2007.271. 24. Morris G, Conner LM; Assessment of accuracy, fix success rate, and use of estimated horizontal position error (EHPE) to filter inaccurate data collected by a common commercially available GPS logger. Plos One 2017;12(11). doi: 10.1371/journal.pone.0189020.

25. R Core Team; R: A Language and Enviroment for Statistical Computing. Vienna, Austria: R Foundation for Statistical Computing, 2016.

266 26. Levitz S, Standley CJ, Adriko M, et al.; Environmental epidemiology of intestinal schistosomiasis and genetic diversity of Schistosoma mansoni infections in snails at Bugoigo village, Lake Albert. Acta Tropica 2013;128(2):284-291. doi: 10.1016/j.actatropica.2012.10.003. 27. Rollinson D, Knopp S, Levitz S, et al.; Time to set the agenda for schistosomiasis elimination. Acta Tropica 2013;128(2):423-440. doi: 10.1016/j.actatropica.2012.04.013.

\section{Figure Legend}

274 Figure 1. A. The S. haematobium egg count frequency for the 21 individuals found positive at the time of community resurvey; B. Plot of water contact events over 24 hour period for mothers $(n=12)$ and PSAC ( $n=12)$, the black lines denote average with $95 \%$ Cls others for mothers $[27.4(-1.3,56.1)]$ and PSAC $[14.1(8.6,19.7)]$ since our sample size was < 30 instead of using the formula of $1.96 *$ Standard error, $2.201 *$ standard error (11 degrees of freedom) was used. No significant difference was found between water contact events for mother and children groups ( $P=0.34$, paired $t$-test $)$ evidencing similar water contact levels; $\mathbf{C}$. GPS co-ordinates of individuals over a 48-hour period stratified by $S$. haematobium infection status and age with different colours representing individuals: (i) not infected PSAC ( $n=11)$; (ii) infected PSAC ( $n=1)$; (iii) uninfected mothers ( $n=10)$; and (iv) infected mothers $(n=2)$. The 
284 S. haematobium infected mothers (M1 and M2) and child (X4) from the GPS study are 285 identified in plots $A$ and $B$. 
288

289

10 I P a g e 
Table 1. Characteristics of individuals enrolled in community-wide parasitological survey $(n=179)$ and subset of mother and PSAC pairs in GPS waterexposure study $(n=24)$.

\begin{tabular}{|c|c|c|c|c|c|}
\hline Characteristic & Total & PSAC & SAC & Adults & P-value ${ }^{b}$ \\
\hline \multicolumn{6}{|c|}{ Community baseline demographics } \\
\hline Number of individuals & 180 & 21 & 55 & 104 & \\
\hline Female, $\mathrm{n}(\%)$ & $102(57.3)$ & $11(52.4)$ & $32(60.4)$ & $59(56.7)$ & 0.808 \\
\hline Age, mean (range) (years) & $26.0(1-84)$ & $3.48(1-6)$ & $11.51(7-16)$ & $38.15(17-84)$ & $<0.001$ \\
\hline \multicolumn{6}{|l|}{ Community parasitology } \\
\hline $\begin{array}{l}\text { S. haematobium positive, } \mathrm{n}(\%) \\
{[95 \% \mathrm{Cl}]}\end{array}$ & $\begin{array}{l}22(12.2) \\
{[7.8,17.9]}\end{array}$ & $\begin{array}{l}2(9.5) \\
{[1.2,30.4]^{c}}\end{array}$ & $\begin{array}{l}7(12.7) \\
{[5.3,24.5]}\end{array}$ & $\begin{array}{l}13(12.5) \\
{[6.8,20.4]}\end{array}$ & 0.922 \\
\hline \multicolumn{6}{|l|}{ Infection intensity, $\mathrm{n}(\%)$} \\
\hline No eggs & $158(87.8)$ & $19(90.5)$ & $48(87.3)$ & $91(87.5)$ & 0.073 \\
\hline $1-50$ eggs $/ 10 \mathrm{ml}$ & $21(11.7)$ & $1(4.8)$ & $7(12.7)$ & $13(12.5)$ & \\
\hline$>50$ eggs $/ 10 \mathrm{ml}$ & $1(0.6)$ & $1(4.8)$ & $0(0.0)$ & $0(0.0)$ & \\
\hline \multicolumn{6}{|c|}{ Mother and PSAC pair baseline demographics } \\
\hline Number of individuals & 24 & 12 & & 12 & \\
\hline Female, n (\%) & $20(83.3 \%)$ & $8(66.6)$ & & $12(100.0)$ & 0.1 \\
\hline Age, mean (range) (years) & $16.23(0.5-52)$ & $2.62(0.5-6)$ & & $29.83(20-52)$ & $<0.001$ \\
\hline \multicolumn{6}{|c|}{ Mother and PSAC pair parasitology } \\
\hline $\begin{array}{l}\text { S. haematobium positive, } \mathrm{n}(\%) \\
{[95 \% \mathrm{Cl}]}\end{array}$ & $\begin{array}{l}3(12.5) \\
{[2.7,32.4]}\end{array}$ & $\begin{array}{l}1(8.3) \\
{[0.2,38.5]}\end{array}$ & & $\begin{array}{l}2(16.7) \\
{[2.1,48.4]}\end{array}$ & 1 \\
\hline
\end{tabular}

a Determined by filtration and microscopy egg-detection in $10 \mathrm{ml}$ urine sample.

${ }^{b} \mathrm{P}$ value is the difference between groups by Fisher exact test or Student's t test.

"Lightly infected PSAC was from GPS study ("X4") and the heavily infected PSAC was from the community cohort (male, aged 4 years). 
Thank you for considering our manuscript and allowing us to respond to the referees. Please find our comments/amendments below.

\section{Reviewer \#1:}

This manuscript illustrates the use of GPS data loggers to measure rates of contact with water bodies among pre-school age children and their mothers in a Cameroonian community where schistosomiasis is endemic. This is a useful method for quantifying potential exposure to schistosomes and it should certainly be adopted more widely to better understand exposure among different demographic groups and to better understand individual exposure heterogeneity. This makes the pilot study interesting, primarily from a methodological standpoint. However, because this is a pilot with a small sample size (24 individuals) and no comparator groups, the results are fundamentally fairly limited in scope and some of the interpretation of the data and subsequent conclusions are overstated. The paper needs to better reflect that this is an illustrative pilot of how GPS dataloggers can be used to quantify contact patterns with water bodies (likely related of exposure to schistosomes) and temper some of the excessively strong conclusions.

\section{Thank you for the comments we have toned down excessively strong conclusions with} modifications throughout and drawn attention to the novelty of the GPS methodology.

Specific comments are below:

1) Abstract, line 24, "In future, PSAC and mothers should be included in both surveillance and control interventions for urogenital schistosomiasis in general." This strong conclusion is not reflective of the data generated from this pilot study. Only the water contact patterns of 12 PSAC and 12 mothers were measured, with no comparator groups from other population demographics. It is not surprising that "...certain individuals had substantive water contact" (line 23). The most appropriate demographic group(s) for surveillance and targeting for the control or elimination of schistosomiasis depends on the relative contact patterns and contributions to transmission among demographic groups. For example, it is quite conceivable that school-age children (SAC) have greater potential exposure (water contact) to schistosomes than PSAC or mothers - in which case they may be the best target group for surveillance (and control). There is a perfectly reasonable debate on whether targeting only SAC is sufficient to control or eliminate schistosomiasis but, in the absence of comparator demographic groups, the data presented here do not provide substantive evidence either way. It cannot be concluded that because water contacts are identified among PSAC and mothers they should necessarily be targeted above other demographic groups for surveillance and control.

In this study we did not have comparator groups across major demographical groups but in a previous study in East Africa we did (see Seto et al. Patterns of intestinal schistosomiasis among mothers and young children from Lake Albert, Uganda: water contact and social networks inferred from wearable global positioning system dataloggers. Geospatial Health 2012;7(1):1-13). In this study we recorded levels for PSAS, SAC, Mothers and Adult Men and also showed that the water contact levels of PSAC and SAC were similar but at different times of the day. More broadly although there is about twofold less water contact per day at Barombi Kotto 
(Cameroon) than in Bugoigo (Uganda), the relative level of water contact in PSAC to mothers shows that they are similar as well (i.e. no signficiant difference). We have modified the text to discuss this point a bit more.

2) Materials \& Methods, line 70, "6 pairs were assigned into two groups to wear GPS dataloggers...". The rationale for dividing the PSAC-mother pairs into two groups is not very clear. Could the Authors give some more detail on this?

This was due to limited number of data loggers (only 13 working loggers in the field due to technical issues with computer-iGOTU interface). We therefore had to use them in 2 rotations, each time using 12 individuals/loggers, which was sufficient to get a snap shot but of course if we had more loggers or greater time in the field we would have collected additional information..

3) Results and Discussion, line 117, "They should be routinely included in disease surveillance and considered for regular treatment with the soon to be deployed paediatric PZQ formulation (12)." This interpretation is beyond the scope of the presented data. The rationale for interventions to target SAC (and omit PSAC and adults) is not based on a presumption that PSAC are unexposed to infection. Rather, it is because SAC tend to harbor the bulk of the parasite population and thus contribute the most to transmission (it is also logistically efficient to reach them through the schools infrastructure). The idea is that the wider population will be protected from infection by the disproportionate reductions in transmission that are achieved by targeting SAC and thus, ultimately, that the disease will be controlled (or eliminated) without requiring community-wide. Whether this approach is best suited to meet current control and elimination goals is actively debated, and there are certainly strong arguments that PSAC and adults should be included in treatment programmes. But the water contact data presented here do not particularly support this argument, especially since only $1 / 20$ PSAC was found to be infected. Again, just because PSAC and mothers are contacting water bodies does not necessarily mean that they are particularly important drivers of transmission or have a higher risk of infection than other demographic groups. To make this conclusion, one would need contact data from the wider population.

\section{Agreed, we have toned down this but called for further studies in other countries to quantify these levels and also assess roles in transmission. This is perhaps more important in locations that are striving for elimination and any infected case that contaminates the environment should be managed.}

4) Results and Discussion, line 121, "these individuals are clear outliers in their level of water contact (4.6, 166.1, and 31.8 for "M1", "M2", and "X1" [sic], respectively)." Looking at the box and whisker plot in Figure 1, panel $B$, it is clear that only $M 2$ is an obvious outlier. It is perhaps also worth the Authors commenting on the low egg count in individual M2 despite the high contact with water. I assume that the quoted "X1" individual is a typographical error and the Authors mean "X4" (as identified in Figure 1)?

We have redrawn this part of the figure with a new plot.

1. Yes typo - should be " $\mathrm{X} 4$ " 
2. Outliers $-M 1$ and $X 4$ both are outside the $95 \%$ confidence intervals for the mean water contact for mothers and PSAC, respectively. However, the current box and whisker plot show quantiles - and only M1 is an outlier of the $75 \%$ quantiles - the revised plot depicts the mean and 95\% Cis, with M1 and X4 shown as outliers.

5) Results and Discussion, line 131, "Another interesting facet revealed by the GPS dataloggers is the similar geospatial pattern of water contact between the two groups which illustrate that PSAC frequently accompany their mothers to the same locations...." Would the Authors not expect that PSAC would frequently accompany their mothers to the same locations? This result seems more confirmatory of a priori expectations than anything else.

It depends on the mother child pair and likely the location and the chore/tasks they are doing. For example, it is not uncommon for children from different guardians to intermingle such that the exact spatial relationship between pairs is 'scrambled'. It does not appear to be so in this instance so is worth a comment.

6) Results and Discussion, line 137, "as a cost-effective measure, it would be sensible to apply focal molluscicides at this location rather than elsewhere, to have highest impact upon removal of infected snail hosts". This is an interesting idea, but again, I feel that this statement should be phrased in a more circumspect manner. Whether such an approach would be cost-effective would depend on a number of factors, including the cost of identifying likely sites of exposure prior to treatment (compared to a more blanket mollusciciding approach) and the infection level of snails in these locations compared to other locations (that are perhaps more frequented by other demographic groups).

We have amended the text and we simply wished to raise the importance of these data with speculation for better targeting of control in future. With the China-Africa initiative, focal mollusciciding is being promoted. It would be a good idea to help synergise these efforts to maximise their impact on the ground.

7) It would be interesting for the Authors to present the water contact data plotted against the parasitological data to explore any relationship. I appreciate that the small sample size will make strong statistical associations unlikely, but the information would still be of interest to the reader.

We have conducted plots as suggested (see below) but the relationship is not so clear hence we prefer to use the revised Figure B plot. The LHS plot is binary plot of infection with water contact, then the RHS plot is intensity of infection with water contact. As the referee suggests if we have more infected cases (6-10 perhaps) it might have made the relationship a bit clearer. 

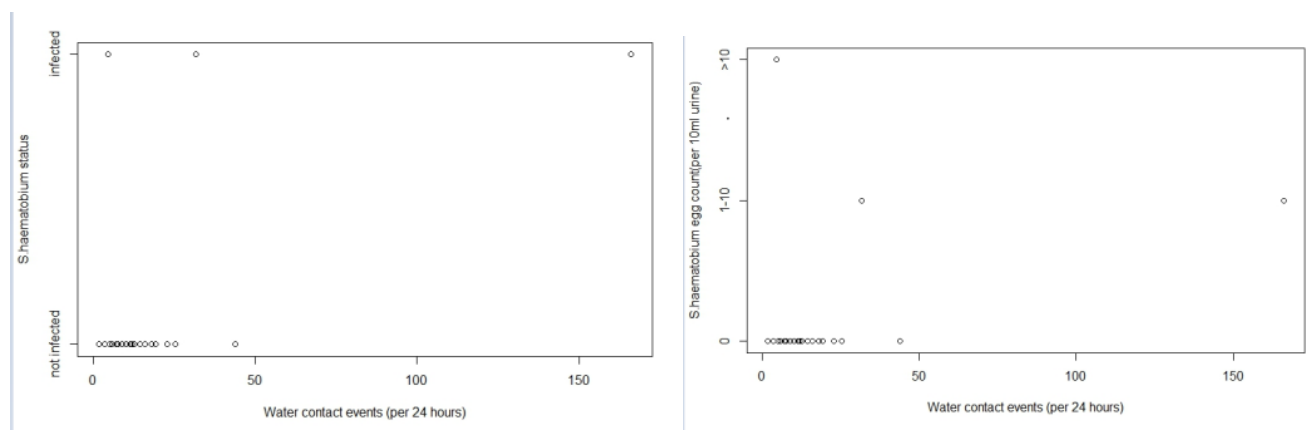

Reviewer \#2:

This is an interesting paper.

The importance of including PSAC and their mothers in control efforts seems clear. Also the use of the datalogger seems an asset, although some issues need clarification.

Some more info on the epidemiology would be useful. Are they waterpumps and pit latrines on the island or on the shore? Are people aware of the risk of contracting schisto, i.e. has health education been given? What do people do for a living? Figure $C$ seems to suggest that people cross the lake to and from the island. Is crossing the lake to the shore a daily activity and for what purpose? Do they wade through the water or, this being a crater lake, is it too deep and do they use boats? How is this counted in terms of exposure?

\section{Thank you for these insights, these are good questions and we have put some background information in the discussion to give a better local insight into the participant activities.}

The section on water exposure measurement does not read well. It would be good if you could explain the accuracy of the measurement better as the number of watercontacts seems very high; on average 27 in 24 hours (in fact between 05:00 and 21:00). How do you know if people are on the beach/shore along the shoreline, or actually in the water? What is the actual time spent during these watercontacts (give range for example); and to what activities do they correspond (for PASC more obvious - playing probably- than for the mothers).

\section{Referring methods we have changed.}

A zone was developed around the lakeshore of the island, $10 \mathrm{~m}$ into the lake and $5 \mathrm{~m}$ into the shore, a conservative assessment of the positional accuracy of the i-gotU-120 data loggers based on previous observations (24). A water contact event was defined as a GPS location recording within a defined lakeshore geospatial buffer zone around the island circumference: a conservative assessment of the positional accuracy of the i-gotU data loggers (24). As the GPS loggers recorded location at 1 minute intervals, each water contact 
event is analogous to 1 minute spent in the geospatial buffer zone and could be tallied and compared between individuals and groups. 
1 A pilot study Uusing wearable global position system (GPS) data loggers to compare water

2 contact levels patterns: Schistosoma haematobium infection in pre-school-aged children

3

4

\section{(PSAC) and their mothers at Barombi Kotto, Cameroon}

Grace Macklin ${ }^{\mathrm{a},+}$, Michelle C. Stanton ${ }^{\mathrm{b}}$, Louis Albert Tchuem-Tchuentéc, J. Russell Stothard ${ }^{\mathrm{a},{ }^{*}}$ ${ }^{a}$ Department of Parasitology, Liverpool School of Tropical Medicine, Liverpool, UK; ${ }^{b}$ Faculty of Health and Medicine, Lancaster University, Lancaster, UK; ' Centre for Schistosomiasis \& Parasitology, Yaoundé, Cameroon

+ present address: London School of Hygiene and Tropical Medicine, Keppel Street, London WC1E 7HT, UK;

* Corresponding author: Tel: +44 151 7053724; E-mail: russell.stothard@Istmed.ac.uk

\section{Abstract}

Barombi Kotto, Cameroon serves as a reference location for assessing intervention strategies against Schistosoma haematobium. As part of a pilot study, the whole community was treated with praziquantel, inclusive of pre-school-aged children (PSAC) and their mothers. One vear later egg-patent infections were reassessed and water contact patterns of 12 pairs of PSAC and their mother were measured with global position system (GPS) dataloggers. A substantial reduction in general infection prevalence, from $44.8 \%$ to $11.7 \%$, was observed but certain PSAC and mothers continued to have egg-patent infections. Analysis of GPS data demonstrated similar water contact levels between child and mother groups, although certain individuals were numerical outliers. This study shows the potential of GPS dataloggers to clarify the at-risk status of PSAC and mothers.

Barombi Kotto, Cameroon serves as a reference location for assessing intervention strategies

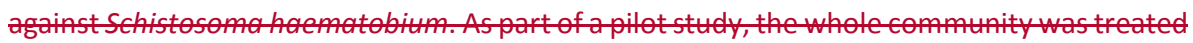
with praziquantel, inclusive of pre-school-aged children (PSAC) and their mothers. One year tater ofo patent infections reassessed and water contact patterns of 12 pairs of PSAC and their mother were measured with wearable global position system (GPS) dataloggers. The cross-sectional resurvey revealed a substantial reduction in general prevalence, from $44.8 \%$ to $11.7 \%$, but PSAC continued to be infected. Analysis of GPS data demonstrated the 1| P a g e 
33 similar at-risk status of child and mother groups, with certain individuals having substantive

34 water contact. In future, PSAC and mothers should be included in both surveillance and

35 control interventions for urogenital schistosomiasis in general.

36

Keywords

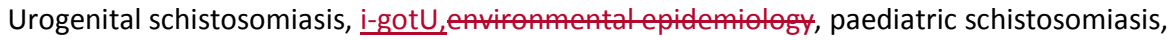
female genital schistosomiasis, praziquantel 


\section{Introduction}

Urogenital schistosomiasis is an important waterborne disease, caused by infection with the blood fluke Schistosoma haematobium, and common in many parts of sub-Saharan Africa (1). In Cameroon, for example, there is a national control programme active in the distribution of praziquantel $(P Z Q)$ to school-aged children $(S A C)(2,3)$. However, in the move towards local interruption of schistosome transmission, the programme is developing new tactics of control (4) and has benefited from recent bilateral support from China in snail control and environmental surveillance (5) as well as from UK to expand access of interventions (6).

Overlooked for too long, expanding access of PZQ to pre-school-aged children (PSAC) and their mothers is attracting increasing attention $(7,8)$. It has been shown elsewhere that these groups can be patently infected (9-12) and alongside SAC, may contribute towards schistosome transmission but but t_their-_water contact(s) is rarely measured and hence the role of PSAC in sustaining local transmission remains speculative $(13,14)$. As a pilot investigation of expanded access to praziquantel treatment, in June 2016 Campbell et al. undertook a detailed cross-sectional epidemiological and malacological survey at Barombi Kotto, Cameroon (15). Barombi Kotto is well-known crater lake and is of significant international interest as a longstanding focus of urogenital schistosomiasis (16-18). Before treating all community members with $\mathrm{PZQ}$, Campbell et al. noted that a quarter of PSAC had egg-patent infections. Furthermore, and adult women had raised signs and symptoms of female genital schistosomiasis (FGS), the latter is of growing international concern $(13,19)$. Environmental water contact is very common across the community, for example, bathing, washing and other domestic chores are typically performed on the immediate shoreline of the island while potable water is collected from a local stream which is only accessible by canoe (15). The water contact levels of both PSAC and their mothers, however, remained to be determined and compared.

To shed fresh light on the at-risk status of PSAC and their mothers, using wearable global position system (GPS) data loggers, we attempted to measure and compare the water contact patterns of PSAC and their mothers $(20,21)$. Furthermore, we hoped to pinpoint water contact sites ${ }_{L}$-measuringe putative immersion times $S_{L}$ on the Barombi Kotto crater lake shoreline as baseline information for future interventions. 


\section{Study location and parasitological examination}

This parasitological resurvey and GPS study was conducted in June 2017 in the community on the central island of Barombi Kotto crater lake, where some 375 people are permanent residents. Study protocols were approved by the Liverpool School of Tropical Medicine Research Ethics Committee and the Cameroon National Ethical Committee of Research for Human Health. Participation involved obtaining written informed consent from mothers and their PSAC before deployment of the GPS datalogger. A total of 179 individuals (20 PSAC, 55 SAC and 104 adults) underwent a parasitological reinspection where each provided a $10 \mathrm{ml}$ urine sample which was filtered and stained with Lugol's iodine to visualise S. haematobium eggs by microscopy as described previously (15). All participants found infected with schistosomiasis were offered and observed to take praziquantel treatment (40 mg/kg)

\section{Water exposure assessment}

A subset of 12 mothers and PSAC pairs were randomly selected, then 6 pairs were assigned into two groups to wear the GPS dataloggers (i-gotUGOTU-120, Mobile Action, UK; dimension $44.5 \times 28.5 \times 13 \mathrm{~mm}$, weight $20 \mathrm{~g}$ ) over a 48-hour period on two occasions. The dataloggers were worn on the arm or wrist using a custom made elastic strap (20). The dataloggers were configured to record GPS location and velocity at 1_-minute intervals during waking hours (05:00-21:00). Analysis of GPS data was conducted in QGIS (22) and filtered per the velocity filtering method (23). A zone was developed around the lakeshore of the island, $10 \mathrm{~m}$ into the take and $5 \mathrm{~m}$ into the shore, a conservative assessment of the positional accuracy of the iGOTU 120 data logrers based on previous observations (24). To quantify water contact events a cumulative total was calculated based on the 1 minute interval recording, for example, each water contact event is analogous to 1 minute spent in the geospatial buffer zone. A zone was developed around the lakeshore of the island, $10 \mathrm{~m}$ into the lake and $5 \mathrm{~m}$ into the shore, a conservative assessment of the positional accuracy of the i-gotU-120 data loggers based on previous observations (24). A water contact event was defined as a GPS location recording within a defined lakeshore geospatial buffer zone around the island circumference: a conservative assessment of the positional accuracy of the i-gotU data loggers (24). As the GPS loggers recorded location at 1 minute intervals, each water contact 
event is analogous to 1 minute spent in the geospatial buffer zone and could be tallied and compared between individuals and groups.

\section{Statistical analysis}

Statistical analysis was performed using the R statistical software (25). Prevalence of schistosomiasis was calculated with $95 \%$ binomial confidence intervals (95\% Cls) with correction for samples of $n<30$. The track logs of each GPS unit were plotted and overlaid against a base map of Barombi Kotto shoreline to identify travel patterns on and off the island.

\section{Results and Discussion}

\section{$<$ please insert table 1 here $>$}

The characteristics of the study population and infection status is shown in Table 1. In June 2017 , the overall prevalence of egg-patent infection was $11.7 \%(95 \% \mathrm{Cl} 7.0$ - 17.0) with only 1 infection of heavy intensity encountered albeit in a PSAC. The epidemiological survey undertaken by Campbell et al. one year previously observed a much higher egg-patent prevalence of $40.1 \%$ (24.6\% in PSAC, 51.3\% in SAC and 44.9 in adults). The overall reduction across all demographic groups, see Table 1 , is most likely due to the community-wide PZQ treatment.

\section{$<$ please insert Figure 1 here $>$}

Twelve PSAC and mother pairs were randomly selected and assigned into two groups of 6 pairs, to wear the GPS dataloggers on two locations. In total, there were 3 individuals with egg-patent infections ( 2 mothers and 1 PSAC) and their intensities of egg-patent infections against the sampled population is shown in Figure 1A. The water contact levels of the 12 mother and child pairs for the 48 hour period is shown in Figure $1 \mathrm{~B}$. This documents a high level of water contact events (reaching up to 166 water contact events in 24 hours) in both mothers and PSAC on the Barombi Kotto shoreline. As data points were recorded at 1ene minute intervals, the number of water contact events can be interpreted as the time 
spent in water contact which would likely positively correlate with actual bodily immersion or skin contact with lake shore water.

The average number of water contact events in 24 hours were 27.4 [95\% Cls: $-1.8 \underline{3}$, 5zㅁ. $9 \underline{1}$ ] for mothers and 14.1 [95\% Cls: $\underline{8} 9 . z \underline{5}, 19.1 \underline{7}]$ for PSAC, with no significant difference between the two groups, Figure 1B. These findings have important implications in that water contact levels of PSAC should not to be overlooked and follow similar levels to that observed in Uganda (20). In Uganda PSAC were observed to spend on average half an hour on the shoreline of Lake Albert and were clearly shown to be aking at-risk vulnerable group not only to first infection but also and in this instance, as evidenced here in Barombi Kotto, re-infection (10). Our study shows the potential of GPS data logging technology to clarify their at-risk status which should assist in better infection surveillance and control of urogenital schistosomiasis in general as well as They should be routinely included in disease surveillance and considered for regular access to treatment with the soon to be deployed paediatric PZQ formulation (12).

Upon more detailed inspection of individual water contact patterns, whilst our GPS sample of two infected mothers ("M1", "M2") and one infected PSAC ("X4") was too small to determine a precise relationship between water exposure and infection status, these individuals weare clear numerical outliers in their level of water contact $(4.6,166.1$, and 31.8 for " $\mathrm{M} 1$ ", "M2" and "X $1 \underline{4}$ ", respectively). The the latter two lying far outside the confidence intervals for PSAC and mother averages, and demonstrates the importance of individual variation in exposure and likely contamination, Figure 1B. Indeed, it is very plausible that the water contact behaviours of " $\mathrm{M} 2$ " and " $\mathrm{X} 4$ " could classify them as 'raised-spreaders' who should be specifically targeted for increased frequency of treatment(s) alongside and behavioural change interventions. It remains to be seen, especially-if these individuals $\forall$ play more pivotal roles than others in facilitating and sustaining local transmission_of schistosomes.

Another interesting facet revealed by the GPS dataloggers is the similar geospatial pattern of water contact between the two groups which illustrate that PSAC frequently accompany their mothers to the same locations, Figure $C$. This is also consistent with other studies using questionnaires $(13,21)$. Furthermore, observed water contacts were largely coclustered on the South-West lakeshore of the island, notably an area where activities of the 6 | P a g e 
3 infected cases were concentrated. Micro-spatial heterogeneity of schistosome transmission has been described elsewhere $(26,27)$ and is further evidenced here, Figure 1D. In the future context of interruption of schistosome transmission $(14,27)$, as a cost-effective measure, it would be sensible to apply focal molluscicides at this location rather than elsewhere, to have highest impact upon removal of infected snail hosts.

Our findings document that GPS dataloggers are an accepted method of measuring water exposure in PSAC and their mothers and directly compare environmental risk of schistosomiasis exposure. We suggest that in future the water contact levels of these two demographic groups should be integrated into control strategies for elimination in Cameroon and elsewherefurther investigated. The wearable GPS technology is also of value to identify putative transmission foci for spatial targeting of interventions.

\section{Authors Contributions}

JRS and MS conceived the study; GM, MS, LATT and JRS designed the study protocol; GM carried out the field work; GM and MS carried out the analysis and interpretation of these data. GM and JRS drafted the manuscript with LATT and MS critically revising. All authors read and approved the final manuscript.

\section{Funding}

GM is in receipt of a Scholarship from the Medical Research Council, United Kingdom. This study was additionally funded as part of the COUNTDOWN research programme consortium, by the Research and Evidence Division of the Department for International Development, UK. The funders had no role in study design, decision to publish, or preparation of the manuscript.

\section{Acknowledgments}

We are grateful to Drs James LaCourse and Martyn Stewart, LSTM who helped with local fieldwork in Cameroon, as well as, the local community and field team volunteers at Barombi Kotto.

\section{Competing interests}

None declared

\section{Ethical approval}

7| P a g e 
The study was approved by the Liverpool School of Tropical Medicine and the Cameroon National Ethical Committee of Research for Human Health.

\section{References}

1. Colley DG, Bustinduy AL, Secor WE, et al.; Human schistosomiasis. Lancet 2014;383(9936):2253-64. doi: 10.1016/S0140-6736(13)61949-2.

2. Tchuente LAT, N'Goran EK; Schistosomiasis and soil-transmitted helminthiasis control in Cameroon and Cote d'Ivoire: implementing control on a limited budget. Parasitology 2009;136(13):1739-1745. doi: 10.1017/s0031182009005988.

3. Tchuente LAT, Noumedem CD, Ngassam P, et al.; Mapping of schistosomiasis and soil-transmitted helminthiasis in the regions of Littoral, North-West, South and South-West Cameroon and recommendations for treatment. Bmc Infectious Diseases 2013;13. doi: 10.1186/1471-2334-13-602.

4. Tchuente LAT, Rollinson D, Stothard JR, et al.; Moving from control to elimination of schistosomiasis in sub-Saharan Africa: time to change and adapt strategies. Infectious Diseases of Poverty 2017;6. doi: 10.1186/s40249-017-0256-8.

5. Xu J, Yu Q, Tchuente LAT, et al.; Enhancing collaboration between China and African countries for schistosomiasis control. Lancet Infectious Diseases 2016;16(3):376-383. doi: 10.1016/s1473-3099(15)00360-6.

6. Stothard JR, Kabatereine NB, Archer J, et al.; A centenary of Robert T. Leiper's lasting legacy on schistosomiasis and a COUNTDOWN on control of neglected tropical diseases. Parasitology 2017;144(12):1602-1612. doi: 10.1017/s0031182016000998.

7. Bustinduy AL, Friedman JF, Kjetland EF, et al.; Expanding Praziquantel (PZQ) Access beyond Mass Drug Administration Programs: Paving a Way Forward for a Pediatric PZQ Formulation for Schistosomiasis. Plos Neglected Tropical Diseases 2016;10(9). doi: 10.1371/journal.pntd.0004946.

8. Lo NC, Addiss DG, Hotez PJ, et al.; A call to strengthen the global strategy against schistosomiasis and soil-transmitted helminthiasis: the time is now. Lancet Infectious Diseases 2017;17(2):E64-E69. doi: 10.1016/s1473-3099(16)30535-7.

9. Bustinduy AL, Stothard JR, Friedman JF; Paediatric and maternal schistosomiasis: shifting the paradigms. British Medical Bulletin 2017;123(1):115-125. doi: 10.1093/bmb/ldx028.

10. Mduluza T, Mutapi F; Putting the treatment of paediatric schistosomiasis into context. Infectious Diseases of Poverty 2017;6. doi: 10.1186/s40249-017-0300-8.

11. Stothard JR, Sousa-Figueiredo JC, Betson M, et al.; Schistosomiasis in African infants and preschool children: let them now be treated! Trends in Parasitology 2013;29(4):197205. doi: 10.1016/j.pt.2013.02.001.

12. Reinhard-Rupp J, Klohe K; Developing a comprehensive response for treatment of children under 6 years of age with schistosomiasis: research and development of a pediatric formulation of praziquantel. Infectious Diseases of Poverty 2017;6. doi: 10.1186/s40249017-0336-9.

13. Poole H, Terlouw DJ, Naunje A, et al.; Schistosomiasis in pre-school-age children and their mothers in Chikhwawa district, Malawi with notes on characterization of schistosomes and snails. Parasites \& Vectors 2014;7. doi: 10.1186/1756-3305-7-153.

14. Stothard JR, Campbell SJ, Osei-Atweneboana MY, et al.; Towards interruption of schistosomiasis transmission in sub-Saharan Africa: developing an appropriate

$8 \mid$ Pag e 
environmental surveillance framework to guide and to support 'end game' interventions. Infectious Diseases of Poverty 2017;6. doi: 10.1186/s40249-016-0215-9.

15. Campbell SJ, Stothard JR, O'Halloran F, et al.; Urogenital schistosomiasis and soiltransmitted helminthiasis (STH) in Cameroon: An epidemiological update at Barombi Mbo and Barombi Kotto crater lakes assessing prospects for intensified control interventions. Infectious Diseases of Poverty 2017;6. doi: 10.1186/s40249-017-0264-8. 16. Duke BOL, Moore PJ; Use of a molluscicide in conjunction with chemotherapy to control Schistosoma haematobium at Barombi Lake Foci in Cameroon. 3. Conclusions and costs. Tropenmedizin Und Parasitologie 1976;27(4):505-508.

17. Gonsufotsin J, Tagnizukam D, Moyousomo R, et al.; Ultrasonographic study of urological lesions of urinary bilhariasis in children in Barombi Kotto (Cameroon). Semaine Des Hopitaux 1990;66(13):680-684.

18. Somo RM, Zukam DT, Kouamouo J, et al.; An epidemiological and radiological study of vesical schistosomiasis foci of the Barombi Lakes, Meme Division, Cameroon. Bulletin De La Societe De Pathologie Exotique 1987;80(5):813-822.

19. Christinet V, Lazdins-Helds JK, Stothard JR, et al.; Female genital schistosomiasis (FGS): from case reports to a call for concerted action against this neglected gynaecological disease. International Journal for Parasitology 2016;46(7):395-404. doi: 10.1016/j.ijpara.2016.02.006.

20. Seto EYW, Sousa-Figueiredo JC, Betson M, et al.; Patterns of intestinal schistosomiasis among mothers and young children from Lake Albert, Uganda: water contact and social networks inferred from wearable global positioning system dataloggers. Geospatial Health 2012;7(1):1-13.

21. Stothard JR, Sousa-Figueiredo JC, Betson M, et al.; Investigating the spatial microepidemiology of diseases within a point-prevalence sample: a field applicable method for rapid mapping of households using low-cost GPS-dataloggers. Transactions of the Royal Society of Tropical Medicine and Hygiene 2011;105(9):500-506. doi:

10.1016/j.trstmh.2011.05.007.

22. QGIS Development Team; QGIS Geographic Information System. Open Source Geospatial Foundation, 2009.

23. Seto EYW, Knapp F, Zhong B, et al.; The use of a vest equipped with a global positioning system to assess water-contact patterns associated with schistosomiasis. Geospatial Health 2007;1(2):233-241. doi: 10.4081/gh.2007.271.

24. Morris G, Conner LM; Assessment of accuracy, fix success rate, and use of estimated horizontal position error (EHPE) to filter inaccurate data collected by a common commercially available GPS logger. Plos One 2017;12(11). doi:

10.1371/journal.pone.0189020.

25. R Core Team; R: A Language and Enviroment for Statistical Computing. Vienna, Austria: R Foundation for Statistical Computing, 2016.

26. Levitz S, Standley CJ, Adriko M, et al.; Environmental epidemiology of intestinal schistosomiasis and genetic diversity of Schistosoma mansoni infections in snails at Bugoigo village, Lake Albert. Acta Tropica 2013;128(2):284-291. doi:

10.1016/j.actatropica.2012.10.003.

27. Rollinson D, Knopp S, Levitz $S$, et al.; Time to set the agenda for schistosomiasis elimination. Acta Tropica 2013;128(2):423-440. doi: 10.1016/j.actatropica.2012.04.013. 


\section{Figure Legend}

Figure 1. A. The $S$. haematobium egg count frequency for the 21 individuals found positive at the time of community resurvey; B. Plot of water contact events over 24 hour period for mothers ( $n=12)$ and PSAC ( $n=12)$, the black lines denote average with $95 \% \mathrm{Cls}$ others for mothers [27.4 $(-1.3,56.1)]$ and PSAC $[14.1(8.6,19.7)]$ since our sample size was < 30 instead of using the formula of $1.96 *$ Standard error $2.201 *$ standard error (11 degrees of freedom) was used.Box and whisker plot of the number of water contact events in 24 hours, for whers $(n-12)$ and PSAC $(n-12)$. No significant difference was found between water contact events for mother and children groups $(P=0.34$, paired $t$-test $)$ evidencing similar water contact levels; C. GPS co-ordinates of individuals over a 48-hour period stratified by $S$. haematobium infection status and age with different colours representing individuals: (i) not infected PSAC ( $n=11)$; (ii) infected PSAC ( $n=1)$; (iii) uninfected mothers $(n=10)$; and (iv) infected mothers ( $n=2)$. The $S$. haematobium infected mothers (M1 and M2) and child (X4) from the GPS study are identified in plots A and B. 
305

306

307

11 P a g e 


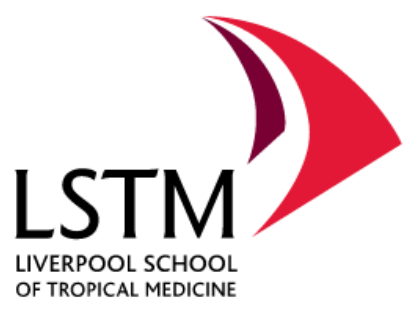

Department of Parasitology

Liverpool School of Tropical Medicine

Liverpool

L3 5QA

$4 / 3 / 2018$

Dear Jonathan,

Please accept my best wishes, and I trust all is well with the RSTM\&H journals.

You may be aware I used to the Honorary Scientific Secretary of the RSMT\&H and have taken an active interest in both journals for many years and have published there since 1995.

Most recently we were delighted with our report in the Transactions, see Al-Shehri (2016) TRSTM\&H) in 2016 and I now have a short paper of sufficient interest to the readership which I would like you to consider.

Our paper is entitled "Using global position system (GPS) data loggers to compare water contact patterns: Schistosoma haematobium infection in pre-school-aged children (PSAC) and their mothers at Barombi Kotto, Cameroon". The work has international value as it looks at two neglected groups in schistosomiasis control - pre-school-aged children and their mothers - as well as implements a novel approach using GPS dataloggers.

The first author Grace Macklin is a hardworking PhD student; she also presented her findings at last year's RIP meeting. After December, I have worked with Grace and the author team to shape her findings into a solid manuscript.

Our manuscript should attract attention not only from those in schistosomiasis control (and paediatric praziquantel initiative) but also has a broader appeal those interested in geospatial epidemiology. The latter has much further application in recording at-risk status of individuals where there is a strong spatial location of transmission.

I look forward to hearing for you and our work has a favourable appraisal. I would recommend the following referees: Professor Clive Shiff (JHSPH); Professor Joanne Webster (RVC); Dr Amadou Garba (WHO); Dr Mark Booth (University of Newcastle).

\section{Professor J.R. Stothard}

Russell.stothard@Istmed.ac.uk 


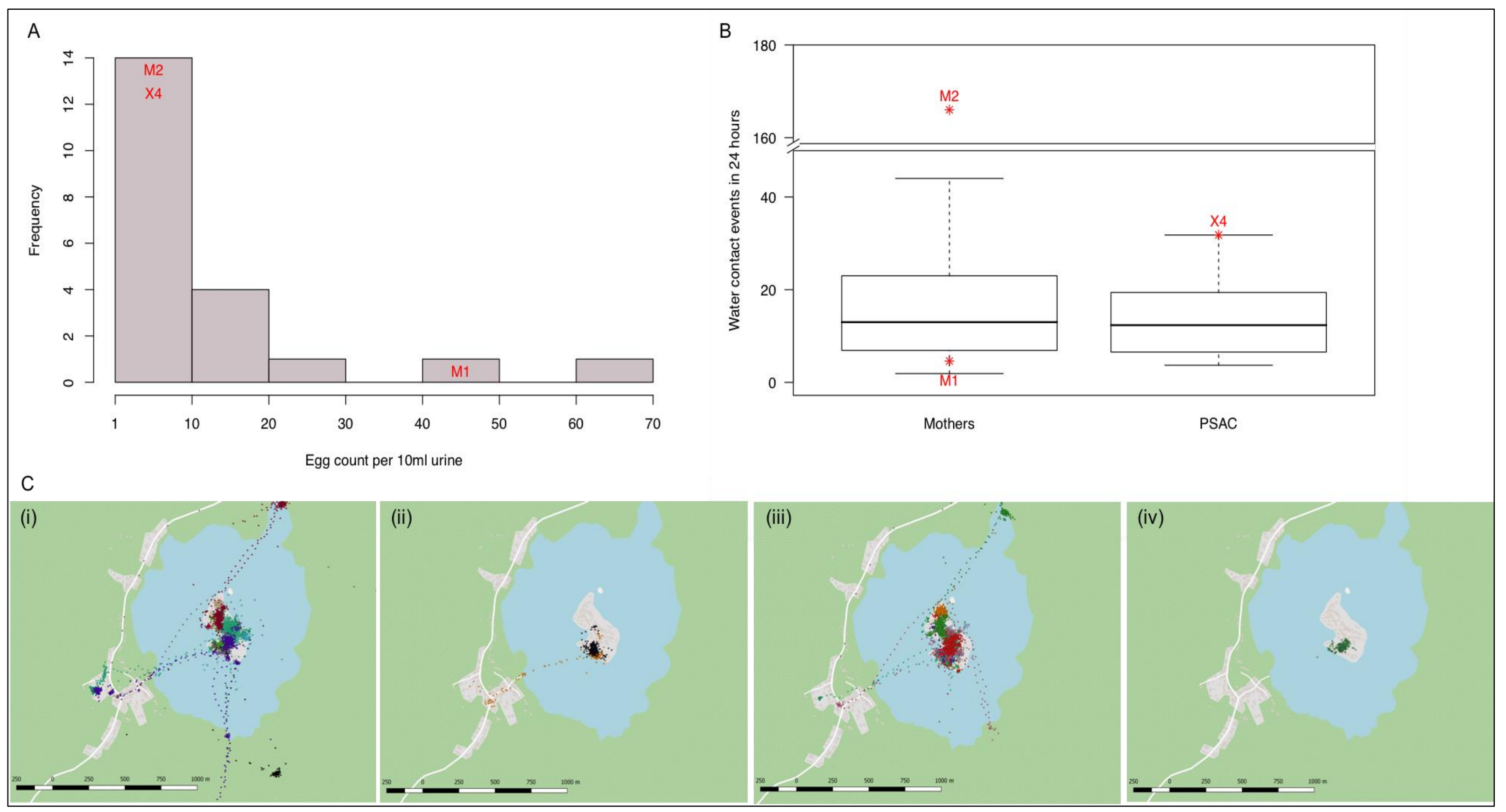

(iii) 
1 Using global position system (GPS) data loggers to compare water contact patterns:

2 Schistosoma haematobium infection in pre-school-aged children (PSAC) and their mothers

3

4

5

6

7

8

10

\section{at Barombi Kotto, Cameroon}

Grace Macklin $^{a,+}$, Michelle C. Stanton ${ }^{b}$, Louis Albert Tchuem-Tchuentéc, J. Russell Stotharda, ${ }^{\text {* }}$

${ }^{a}$ Department of Parasitology, Liverpool School of Tropical Medicine, Liverpool, UK; ${ }^{b}$ Faculty of Health and Medicine, Lancaster University, Lancaster, UK; ${ }^{c}$ Centre for Schistosomiasis \& Parasitology, Yaoundé, Cameroon

+ present address: London School of Hygiene and Tropical Medicine, Keppel Street, London WC1E 7HT, UK;

* Corresponding author: Tel: +44 151 7053724; E-mail: russell.stothard@lstmed.ac.uk

\section{Abstract}

Barombi Kotto, Cameroon serves as a reference location for assessing intervention strategies against Schistosoma haematobium. As part of a pilot study, the whole community was treated with praziquantel, inclusive of pre-school-aged children (PSAC) and their mothers. One year later egg-patent infections were reassessed and water contact patterns of 12 pairs of PSAC and their mother were measured with wearable global position system (GPS) dataloggers. The cross-sectional resurvey revealed a substantial reduction in general prevalence, from $44.8 \%$ to $11.7 \%$, but PSAC continued to be infected. Analysis of GPS data demonstrated the similar at-risk status of child and mother groups, with certain individuals having substantive water contact. In future, PSAC and mothers should be included in both surveillance and control interventions for urogenital schistosomiasis in general.

\section{Keywords}

Urogenital schistosomiasis, environmental epidemiology, paediatric schistosomiasis, female genital schistosomiasis, praziquantel 


\section{Introduction}

Urogenital schistosomiasis is an important waterborne disease, caused by infection with the blood fluke Schistosoma haematobium, and common in many parts of sub-Saharan Africa (1). In Cameroon, for example, there is a national control programme active in the distribution of praziquantel (PZQ) to school-aged children (SAC) $(2,3)$. However, in the move towards local interruption of schistosome transmission, the programme is developing new tactics of control (4) and has benefited from recent bilateral support from China in snail control and environmental surveillance (5) as well as from UK to expand access of interventions (6).

Overlooked for too long, expanding access of PZQ to pre-school-aged children (PSAC) and their mothers is attracting increasing attention $(7,8)$. It has been shown elsewhere that these groups can be patently infected (9-12) and alongside SAC, may contribute towards schistosome transmission but their water contact(s) is rarely measured $(13,14)$. As a pilot investigation of expanded access to praziquantel treatment, in June 2016 Campbell et al. undertook a detailed cross-sectional epidemiological and malacological survey at Barombi Kotto, Cameroon (15). Barombi Kotto is well-known crater lake and is of significant international interest as a longstanding focus of urogenital schistosomiasis (16-18). Before treating all community members with PZQ, Campbell et al. noted that a quarter of PSAC had egg-patent infections and adult women had raised signs and symptoms of female genital schistosomiasis (FGS), the latter is of growing international concern $(13,19)$. The water contact levels of PSAC and their mothers, however, remained to be determined.

To shed fresh light on the at-risk status of PSAC and their mothers, using wearable global position system (GPS) data loggers, we attempted to measure the water contact patterns of PSAC and their mothers $(20,21)$. Furthermore, we hoped to pinpoint water contact sites and measure putative immersion times on the Barombi Kotto crater lake shoreline as baseline information for future interventions.

\section{Methods \& Materials}

\section{Study location and parasitological examination}

This parasitological resurvey and GPS study was conducted in June 2017 in the community on the central island of Barombi Kotto crater lake, where some 375 people are permanent residents. Study protocols were approved by the Liverpool School of Tropical Medicine Research Ethics Committee and the Cameroon National Ethical Committee of Research for 
Human Health. Participation involved obtaining written informed consent from mothers and their PSAC before deployment of the GPS datalogger. A total of 179 individuals (20 PSAC, 55 SAC and 104 adults) underwent a parasitological reinspection where each provided a $10 \mathrm{ml}$ urine sample which was filtered and stained with Lugol's iodine to visualise S. haematobium eggs by microscopy as described previously (15). All participants found infected with schistosomiasis were offered and observed to take praziquantel treatment ( $40 \mathrm{mg} / \mathrm{kg})$

\section{Water exposure assessment}

A subset of 12 mothers and PSAC pairs were randomly selected, then 6 pairs were assigned into two groups to wear the GPS dataloggers (i-GOTU-120, Mobile Action, UK; dimension 44.5 x $28.5 \times 13 \mathrm{~mm}$, weight $20 \mathrm{~g}$ ) over a 48-hour period on two occasions. The dataloggers were worn on the arm or wrist using a custom made elastic strap (20). The dataloggers were configured to record GPS location and velocity at 1 minute intervals during waking hours (05:00-21:00). Analysis of GPS data was conducted in QGIS (22) and filtered per the velocity filtering method (23). A zone was developed around the lakeshore of the island, 10m into the lake and $5 \mathrm{~m}$ into the shore, a conservative assessment of the positional accuracy of the iGOTU-120 data loggers based on previous observations (24). To quantify water contact events a cumulative total was calculated based on the 1 minute interval recording, for example, each water contact event is analogous to 1 minute spent in the geospatial buffer zone.

82

\section{Statistical analysis}

Statistical analysis was performed using the R statistical software (25). Prevalence of schistosomiasis was calculated with $95 \%$ binomial confidence intervals. The track logs of each GPS unit were plotted and overlaid against a base map of Barombi Kotto shoreline.

\section{Results and Discussion}

89

\section{<please insert table 1 here $>$}

The characteristics of the study population and infection status is shown in Table 1. In June 2017 , the overall prevalence of egg-patent infection was $11.7 \%$ (95\% Cl 7.0 - 17.0) with only 
1 infection of heavy intensity encountered albeit in a PSAC. The epidemiological survey undertaken by Campbell et al. one year previously observed a much higher egg-patent prevalence of $40.1 \%$ ( $24.6 \%$ in PSAC, $51.3 \%$ in SAC and 44.9 in adults). The overall reduction across all demographic groups, see Table 1 , is most likely due to the community-wide PZQ treatment.

\section{<please insert Figure 1 here}

Twelve PSAC and mother pairs were randomly selected and assigned into two groups of 6 pairs, to wear the GPS dataloggers on two locations. In total, there were 3 individuals with egg-patent infections ( 2 mothers and 1 PSAC) and their intensities of egg-patent infections against the sampled population is shown in Figure $1 \mathrm{~A}$. The water contact levels of the 12 mother and child pairs for the 48 hour period is shown in Figure 1B. This documents a high level of water contact events (reaching up to 166 water contact events in 24 hours) in both mothers and PSAC on the Barombi Kotto shoreline. As data points were recorded at one minute intervals, the number of water contact events can be interpreted as the time spent in water contact which would likely positively correlate with actual bodily immersion or skin contact with lake shore water.

The average number of water contact events in 24 hours were 27.4 [95\% Cls: $1.8,52.9$ ] for mothers and 14.1 [95\% Cls: $9.2,19.1]$ for PSAC, with no significant difference between the two groups, Figure 1B. These findings have important implications in that water contact levels of PSAC are not to be overlooked and follow similar levels to that observed in Uganda (20) making them a proven at-risk vulnerable group to infection and in this instance re-infection (10). They should be routinely included in disease surveillance and considered for regular treatment with the soon to be deployed paediatric PZQ formulation (12).

Upon more detailed inspection of individual water contact patterns, whilst our GPS sample of two infected mothers ("M1", "M2") and one infected PSAC ("X4") was too small to determine a precise relationship between water exposure and infection status, these individuals are clear outliers in their level of water contact $(4.6,166.1$, and 31.8 for "M1", "M2" and "X1", respectively). The the latter two lying far outside the confidence intervals for PSAC and mother averages, and demonstrates the importance of individual variation in exposure and likely contamination, Figure 1B. Indeed, it is very plausible that the water 
contact behaviours of " $\mathrm{M} 2$ " and "X4" might classify them as 'raised-spreaders' who should be specifically targeted for increased frequency of treatment(s) and behavioural change interventions, especially if they play more pivotal roles than others in facilitating local transmission.

Another interesting facet revealed by the GPS dataloggers is the similar geospatial pattern of water contact between the two groups which illustrate that PSAC frequently accompany their mothers to the same locations, Figure $\mathrm{C}$. This is also consistent with other studies using questionnaires $(13,21)$. Furthermore, observed water contacts were largely coclustered on the South-West lakeshore of the island, notably an area where activities of the 3 infected cases were concentrated. Micro-spatial heterogeneity of schistosome transmission has been described elsewhere $(26,27)$ and is further evidenced here, Figure 1D. In the future context of interruption of schistosome transmission $(14,27)$, as a cost-effective measure, it would be sensible to apply focal molluscicides at this location rather than elsewhere, to have highest impact upon removal of infected snail hosts.

Our findings document that GPS dataloggers are an accepted method of measuring water exposure in PSAC and their mothers and directly compare environmental risk of schistosomiasis exposure. We suggest that these two demographic groups should be integrated into control strategies for elimination in Cameroon and elsewhere. The GPS technology is also of value to identify putative transmission foci for spatial targeting of interventions.

\section{Authors Contributions}

148 JRS and MS conceived the study; GM, MS, LATT and JRS designed the study protocol; GM 149 carried out the field work; GM and MS carried out the analysis and interpretation of these 150 data. GM and JRS drafted the manuscript with LATT and MS critically revising. All authors read and approved the final manuscript.

\section{Funding}

154 GM is in receipt of a Scholarship from the Medical Research Council, United Kingdom. This study was additionally funded as part of the COUNTDOWN research programme consortium, by the Research and Evidence Division of the Department for International Development, UK. The funders had no role in study design, decision to publish, or preparation of the manuscript. 
158

159

160

161

162

163

164

165

166

167

168

169

170

171

172

173

174

175

176

177

178

179

180

181

182

183

184

185

186

187

188

189

190

191

192

193

194

195

196

197

198

\section{Acknowledgments}

We are grateful to Drs James LaCourse and Martyn Stewart, LSTM who helped with local fieldwork in Cameroon, as well as, the local community and field team volunteers at Barombi Kotto.

\section{Competing interests}

None declared

\section{Ethical approval}

The study was approved by the Liverpool School of Tropical Medicine and the Cameroon National Ethical Committee of Research for Human Health.

\section{References}

1. Colley DG, Bustinduy AL, Secor WE, et al.; Human schistosomiasis. Lancet 2014;383(9936):2253-64. doi: 10.1016/S0140-6736(13)61949-2.

2. Tchuente LAT, N'Goran EK; Schistosomiasis and soil-transmitted helminthiasis control in Cameroon and Cote d'Ivoire: implementing control on a limited budget. Parasitology 2009;136(13):1739-1745. doi: 10.1017/s0031182009005988.

3. Tchuente LAT, Noumedem CD, Ngassam P, et al.; Mapping of schistosomiasis and soil-transmitted helminthiasis in the regions of Littoral, North-West, South and South-West Cameroon and recommendations for treatment. Bmc Infectious Diseases 2013;13. doi: 10.1186/1471-2334-13-602.

4. Tchuente LAT, Rollinson D, Stothard JR, et al.; Moving from control to elimination of schistosomiasis in sub-Saharan Africa: time to change and adapt strategies. Infectious Diseases of Poverty 2017;6. doi: 10.1186/s40249-017-0256-8.

5. Xu J, Yu Q, Tchuente LAT, et al.; Enhancing collaboration between China and African countries for schistosomiasis control. Lancet Infectious Diseases 2016;16(3):376-383. doi: 10.1016/s1473-3099(15)00360-6.

6. Stothard JR, Kabatereine NB, Archer J, et al.; A centenary of Robert T. Leiper's lasting legacy on schistosomiasis and a COUNTDOWN on control of neglected tropical diseases. Parasitology 2017;144(12):1602-1612. doi: 10.1017/s0031182016000998.

7. Bustinduy AL, Friedman JF, Kjetland EF, et al.; Expanding Praziquantel (PZQ) Access beyond Mass Drug Administration Programs: Paving a Way Forward for a Pediatric PZQ Formulation for Schistosomiasis. Plos Neglected Tropical Diseases 2016;10(9). doi: 10.1371/journal.pntd.0004946.

8. Lo NC, Addiss DG, Hotez PJ, et al.; A call to strengthen the global strategy against schistosomiasis and soil-transmitted helminthiasis: the time is now. Lancet Infectious Diseases 2017;17(2):E64-E69. doi: 10.1016/s1473-3099(16)30535-7.

9. Bustinduy AL, Stothard JR, Friedman JF; Paediatric and maternal schistosomiasis: shifting the paradigms. British Medical Bulletin 2017;123(1):115-125. doi: 10.1093/bmb/ldx028. 
10. Mduluza T, Mutapi F; Putting the treatment of paediatric schistosomiasis into context. Infectious Diseases of Poverty 2017;6. doi: 10.1186/s40249-017-0300-8.

11. Stothard JR, Sousa-Figueiredo JC, Betson M, et al.; Schistosomiasis in African infants and preschool children: let them now be treated! Trends in Parasitology 2013;29(4):197205. doi: 10.1016/j.pt.2013.02.001.

12. Reinhard-Rupp J, Klohe K; Developing a comprehensive response for treatment of children under 6 years of age with schistosomiasis: research and development of a pediatric formulation of praziquantel. Infectious Diseases of Poverty 2017;6. doi: 10.1186/s40249017-0336-9.

13. Poole H, Terlouw DJ, Naunje A, et al.; Schistosomiasis in pre-school-age children and their mothers in Chikhwawa district, Malawi with notes on characterization of schistosomes and snails. Parasites \& Vectors 2014;7. doi: 10.1186/1756-3305-7-153.

14. Stothard JR, Campbell SJ, Osei-Atweneboana MY, et al.; Towards interruption of schistosomiasis transmission in sub-Saharan Africa: developing an appropriate environmental surveillance framework to guide and to support 'end game' interventions. Infectious Diseases of Poverty 2017;6. doi: 10.1186/s40249-016-0215-9.

15. Campbell SJ, Stothard JR, O'Halloran F, et al.; Urogenital schistosomiasis and soiltransmitted helminthiasis (STH) in Cameroon: An epidemiological update at Barombi $\mathrm{Mbo}$ and Barombi Kotto crater lakes assessing prospects for intensified control interventions. Infectious Diseases of Poverty 2017;6. doi: 10.1186/s40249-017-0264-8. 16. Duke BOL, Moore PJ; Use of a molluscicide in conjunction with chemotherapy to control Schistosoma haematobium at Barombi Lake Foci in Cameroon. 3. Conclusions and costs. Tropenmedizin Und Parasitologie 1976;27(4):505-508.

17. Gonsufotsin J, Tagnizukam D, Moyousomo R, et al.; Ultrasonographic study of urological lesions of urinary bilhariasis in children in Barombi Kotto (Cameroon). Semaine Des Hopitaux 1990;66(13):680-684.

18. Somo RM, Zukam DT, Kouamouo J, et al.; An epidemiological and radiological study of vesical schistosomiasis foci of the Barombi Lakes, Meme Division, Cameroon. Bulletin De La Societe De Pathologie Exotique 1987;80(5):813-822.

19. Christinet V, Lazdins-Helds JK, Stothard JR, et al.; Female genital schistosomiasis (FGS): from case reports to a call for concerted action against this neglected gynaecological disease. International Journal for Parasitology 2016;46(7):395-404. doi: 10.1016/j.ijpara.2016.02.006.

20. Seto EYW, Sousa-Figueiredo JC, Betson M, et al.; Patterns of intestinal schistosomiasis among mothers and young children from Lake Albert, Uganda: water contact and social networks inferred from wearable global positioning system dataloggers. Geospatial Health 2012;7(1):1-13.

21. Stothard JR, Sousa-Figueiredo JC, Betson M, et al.; Investigating the spatial microepidemiology of diseases within a point-prevalence sample: a field applicable method for rapid mapping of households using low-cost GPS-dataloggers. Transactions of the Royal Society of Tropical Medicine and Hygiene 2011;105(9):500-506. doi: 10.1016/j.trstmh.2011.05.007.

22. QGIS Development Team; QGIS Geographic Information System. Open Source Geospatial Foundation, 2009.

23. Seto EYW, Knapp F, Zhong B, et al.; The use of a vest equipped with a global positioning system to assess water-contact patterns associated with schistosomiasis. Geospatial Health 2007;1(2):233-241. doi: 10.4081/gh.2007.271. 
24. Morris G, Conner LM; Assessment of accuracy, fix success rate, and use of estimated horizontal position error (EHPE) to filter inaccurate data collected by a common commercially available GPS logger. Plos One 2017;12(11). doi:

249 10.1371/journal.pone.0189020.

$25025 . \quad$ R Core Team; R: A Language and Enviroment for Statistical Computing. Vienna, 251 Austria: R Foundation for Statistical Computing, 2016.

252 26. Levitz S, Standley CJ, Adriko M, et al.; Environmental epidemiology of intestinal 253 schistosomiasis and genetic diversity of Schistosoma mansoni infections in snails at Bugoigo 254 village, Lake Albert. Acta Tropica 2013;128(2):284-291. doi:

255 10.1016/j.actatropica.2012.10.003.

256 27. Rollinson D, Knopp S, Levitz S, et al.; Time to set the agenda for schistosomiasis 257 elimination. Acta Tropica 2013;128(2):423-440. doi: 10.1016/j.actatropica.2012.04.013.

\section{Figure Legend}

260 Figure 1. A. The S. haematobium egg count frequency for the 21 individuals found positive 261 at the time of community resurvey; B. Box and whisker plot of the number of water contact 262 events in 24 hours, for mothers $(n=12)$ and PSAC $(n=12)$. No significant difference was found 263 between mother and children groups ( $P=0.34$, paired t-test); C. GPS co-ordinates of individuals over a 48-hour period stratified by S. haematobium infection status and age with different colours representing individuals: (i) not infected PSAC ( $n=11$ ); (ii) infected PSAC ( $n=1)$; (iii) uninfected mothers ( $n=10)$; and (iv) infected mothers $(n=2)$. The S. haematobium infected mothers (M1 and M2) and child (X4) from the GPS study are identified in plots A and $B$. 
270

271

272

9| Pag e 
Table 1. Characteristics of individuals enrolled in community-wide parasitological survey $(n=179)$ and subset of mother and PSAC pairs in GPS waterexposure study $(n=24)$.

\begin{tabular}{|c|c|c|c|c|c|}
\hline Characteristic & Total & PSAC & SAC & Adults & P-value ${ }^{b}$ \\
\hline \multicolumn{6}{|c|}{ Community baseline demographics } \\
\hline Number of individuals & 179 & 20 & 55 & 104 & \\
\hline Female, $\mathrm{n}(\%)$ & $101(57.1)$ & $10(50.0)$ & $32(60.4)$ & $59(56.7)$ & 0.723 \\
\hline Age, mean(range) (years) & $26.1(1-84)$ & $3.50(1-6)$ & $11.51(7-16)$ & $38.15(17-84)$ & $<0.001$ \\
\hline \multicolumn{6}{|l|}{ Community parasitology } \\
\hline $\begin{array}{l}\text { S. haematobium positive, } \mathrm{n}(\%) \\
{[95 \% \mathrm{Cl}]}\end{array}$ & $\begin{array}{l}21(11.7) \\
{[0.07,0.17]}\end{array}$ & $\begin{array}{l}1(5.0) \\
{[0.001,0.25]}\end{array}$ & $\begin{array}{l}7(12.7) \\
{[0.05,0.24]}\end{array}$ & $\begin{array}{l}13(12.5) \\
{[0.07,0.20]}\end{array}$ & 0.610 \\
\hline \multicolumn{6}{|l|}{ Infection intensity, $\mathrm{n}(\%)$} \\
\hline No eggs & $158(88.3)$ & $19(95.0)$ & $48(87.3)$ & $91(87.5)$ & 0.032 \\
\hline $1-50$ eggs $/ 10 \mathrm{ml}$ & $20(11.0)$ & $0(0.0)$ & $7(12.7)$ & $13(12.5)$ & \\
\hline$>50$ eggs $/ 10 \mathrm{ml}$ & $1(0.5)$ & $1(5.0)$ & $0(0.0)$ & $0(0.0)$ & \\
\hline \multicolumn{6}{|c|}{ Mother and PSAC pair baseline demographics } \\
\hline Number of individuals & 24 & 12 & & 12 & \\
\hline Female, n (\%) & $20(83.3 \%)$ & $8(66.6)$ & & $12(100.0)$ & 0.1 \\
\hline Age, mean (range) (years) & $16.23(0.5-52)$ & $2.62(0.5-6)$ & & $29.83(20-52)$ & $<0.001$ \\
\hline \multicolumn{6}{|c|}{ Mother and PSAC pair parasitology } \\
\hline $\begin{array}{l}\text { S. haematobium positive, } \mathrm{n}(\%) \\
{[95 \% \mathrm{Cl}]}\end{array}$ & 3 & $\begin{array}{l}1(8.3) \\
{[0.002,0.38]}\end{array}$ & & $\begin{array}{l}2(16.7) \\
{[0.02,0.48]}\end{array}$ & 1 \\
\hline
\end{tabular}

a Determined by filtration and microscopy egg-detection in $10 \mathrm{ml}$ urine sample.

${ }^{b} \mathrm{P}$ value is the difference between groups by Fisher exact test or Student's $\mathrm{t}$ test. 